\title{
Dopamine Cell Transplantation for Parkinson's Disease: The Importance of Controlled Clinical Trials
}

\author{
Curt R. Freed • Wenbo Zhou • Robert E. Breeze
}

Published online: 15 October 2011

(C) The American Society for Experimental NeuroTherapeutics, Inc. 2011

\begin{abstract}
Transplantation of human fetal dopamine neurons into the brain of Parkinson's disease patients started in the late 1980s, less than 10 years after experiments in rats showed that embryonic dopamine neurons from a narrow window of development are suitable for transplantation. For human transplantation, the critical stage of development is 6 to 8 weeks after conception. Because putamen is the basal ganglia structure most depleted of dopamine in Parkinson's disease and because it is the structure most closely mapped to the motor cortex, it has been the primary target for neurotransplantation. The double blind trial conducted at the University of Colorado, Columbia University, and North Shore University is the first controlled surgical trial performed in the field of neurosurgery. Results have shown that transplants of fetal dopamine neurons can survive transplantation without immunosuppression and without regard to the age of the patients. Transplants improved objective signs of Parkinson's disease to the best effects of LDOPA seen preoperatively. Placebo surgery produced no clinical changes. In subjects in whom transplants replaced the need for L-DOPA, the implants replicated the preoperative effects of L-DOPA, including dyskinesias in susceptible patients. Our trial has provided the first controlled evidence that dopamine cell transplants can improve the clinical state of patients with Parkinson's disease.
\end{abstract}

Keywords Fetal dopamine neurons · Putamen - Caudate nucleus · Double-blind trial · Embryonic stem cells $\cdot$ Induced pluripotent stem cells $\cdot$ Immunosuppression

Electronic supplementary material The online version of this article (doi:10.1007/s13311-011-0082-9) contains supplementary material, which is available to authorized users.

C. R. Freed $(\bowtie) \cdot$ W. Zhou $\cdot$ R. E. Breeze

University of Colorado School of Medicine,

Aurora, CO 80045, USA

e-mail: curt.freed@ucdenver.edu

\section{Introduction}

Transplantation of human fetal dopamine neurons into the brains of patients with Parkinson's disease, which started in the late 1980s (less than 10 years after experiments in rats), showed that embryonic dopamine neurons from a narrow window of development (13 to 15 days after conception) are suitable for transplantation $[1,2]$. For human transplantation, the equivalent stage of embryonic development is 6 to 8 weeks after conception [3-5]. All successful allografts of human embryonic tissue into patients with Parkinson's disease have come from tissue in this developmental window. Because putamen is the basal ganglia structure most depleted of dopamine in Parkinson's disease, and because it is the structure most closely mapped to the motor cortex, it has been the primary target for neurotransplantation [6]. Although the caudate nucleus and substantia nigra have also been implanted with dopamine neurons, it is unclear whether cells transplanted into these regions have provided additional beneficial effects in patients with Parkinson's disease.

Until 1993, federal policy would not allow the National Institutes of Health (NIH) to fund research on transplanting human fetal dopamine neurons to humans. When President Clinton overturned that policy, we proposed a controlled clinical trial of transplantation with cell implantation compared to a sham surgical procedure, the first doubleblind trial ever performed in the field of neurosurgery [7]. Although the concept of a double-blind neurosurgical trial was novel and controversial at that time [8], subsequent to our study there have been several double-blind neurosurgical studies for Parkinson's disease either transplanting cells or injecting growth factors [9-11].

While tissues other than human fetal dopamine neurons have been transplanted into Parkinson patients, only human ventral mesencephalic dopamine neurons have produced clinical improvement. For a brief period in the 1980s, 
adrenal chromaffin cells from the patient's own adrenal glands were transplanted into the head of the caudate nucleus. These operations were associated with substantial morbidity and produced no clinical benefit [12].

To get around the extraordinary challenge of recovering ventral mesencephalon from elective abortion of early embryos, efforts have been made to transplant tissue from other species into humans. Dopamine neurons from the fetal pig were approved for both phase I and phase II clinical trials. Despite promising results from a phase I study [13], the double-blind phase II trial showed that patients receiving pig neurons had no clinical benefit compared to sham surgery; both the placebo group and the implanted group had large improvement in their Parkinson's disease scores (not yet published). Although all patients were immunosuppressed with cyclosporine and prednisone to prevent rejection of the porcine xenografts, only a very small number of pig dopamine neurons were found to survive [14]. At the present time, transplantation of cells from other species presents an insurmountable immunological problem. A similar outcome was seen with a manufactured product, Spheramine (Mfg: Titan Pharmaceuticals, South San Francisco, CA), which combined human retinal pigment epithelial cells with a microcarrier support matrix, which was then injected into the putamen. Although results of a phase I open clinical trial were positive [15], the double-blind phase II trial failed to show any benefit.

As noted above, several promising phase I neurosurgical observations have failed to be validated in double-blind trials. This fact draws into question the validity of all neurosurgical therapies for Parkinson's disease that have not been conducted with blinded clinical assessments and concurrent sham surgery controls.

A major problem in evaluating transplants for Parkinson's disease has been the small numbers of patients receiving transplants in most centers. Transplant methods have varied widely between, and even within, individual centers, and case reports of a single patient or a few patients are common. These small numbers coupled with the natural bias in favor of a positive clinical outcome have made it difficult to decide whether any clinical benefit has been achieved.

Because our double-blind clinical trial implanted fetal dopamine neurons into a total of 34 subjects using the same surgical protocol with 20 subjects implanted and 20 receiving sham surgery under double-blind observation, and 14 more implanted in later unblended surgeries using the same transplant method, ours is by far the largest cohort available to determine transplant outcomes. We have found that human fetal dopamine neurons can survive transplantation into the putamen, regardless of the age of the patient and without immunosuppression, and can improve signs of Parkinson's disease as great as the best effects of L-DOPA. We have seen that the dopaminergic effects of transplants cannot be better than the best effects of L-DOPA, and hence they do not improve the "best on" state. Transplants supply dopamine continuously to the putamen and should be viewed as equivalent to a steady-state infusion of L-DOPA. In patients who had preoperative L-DOPA-induced dyskinesias, transplants that developed well enough to replace the need for L-DOPA also led to persistent dyskinesias as part of the continuous "on" state. This review presents the data leading to those conclusions while demonstrating the critical importance of double blind, controlled surgical trials for establishing therapeutic benefit.

\section{Animal Models Guiding Human Neurotransplantation}

Progress toward human neurotransplantation was made possible by the rat model of Parkinson's disease developed by Ungerstedt and Arbuthnott. They showed that unilateral injection of the neurotoxin 6-hydroxydopamine (6-OHDA) into the medial forebrain bundle of rat brain can destroy dopamine neurons in the substantia nigra pars compacta with loss of dopamine nerve terminals in the striatum [16]. Nearly complete destruction of dopamine neurons can be achieved on one side of the brain without damaging other neural systems or dopamine neurons on the contralateral side. Animals maintain nearly normal eating, drinking, and grooming behaviors. When doses of methamphetamine are administered that ordinarily produce stereotypic behavior ( $5 \mathrm{mg} / \mathrm{kg}$ subcutaneously), the unilaterally lesioned animals will begin circling toward their lesioned side at rates as high as $10 \mathrm{rpm}$. Dopamine released asymmetrically from the intact side of striatum drives the circling behavior. Only rats with greater than $95 \%$ unilateral dopamine depletion will circle at these high rates. By contrast, the dopamine agonist apomorphine stimulates the supersensitive dopamine receptors in the denervated striatum and leads to circling in the direction contralateral to the lesion.

This rat model made it possible to explore cell replacement therapies for Parkinson's disease. Cells from a wide developmental range from early embryonic to adult tissue were tested for their ability to survive in brain. Critical experiments by Bjorklund and Stenevi [1] and Perlow and colleagues [2] were the first to show that rat mesencephalic dopamine cells transplanted into the denervated striatum could survive, extend neurites, and stop the circling response to amphetamine as dopamine concentrations were restored.

These early experiments were followed by others which showed that dopamine neurite outgrowth occurred in response to factors produced by the dopamine denervated striatum. Only mesencephalic dopamine neurons will reinnervate the striatum, and transplant growth occurs only after striatum has been denervated. The behavioral effects of transplantation in rats are specific to mesencephalic dopamine cells. Rats transplanted with serotonergic neurons from mesencephalic raphe or dopamine neurons from 
hypothalamus have no improvement in behavior [17, 18]. These experiments showed that innervation of the target is a specific interaction between the appropriate dopamine neuron and factors released from the denervated striatum.

This important principle provides an argument for the safety of dopamine cell transplants, suggesting that a wide range of tissue doses could produce an acceptable behavioral response, because the host striatum would secrete neurotrophic factors until appropriate dopaminergic input was achieved. Because this response is likely to be very local, even confined to individual or small groups of striatal neurons, the model does not assure homogeneous reinnervation by dopamine neurons. Because the grafted dopamine neurons are ectopically placed in the striatum and do not have normal afferents, the dopamine neurons do not receive normal inputs regulating their own firing rates. If those inputs are needed for fully normal control of movement, dopamine cell transplants always will be limited in value.

UItrastructural studies have shown that fetal grafts reinnervate denervated striatum with both host-to-graft and graft-to-host synapses [19]. Dopaminergic nerve terminals synapse on dendritic spines of medium spiny neurons. Transplanted cells synthesize and release dopamine [20]. Grafted dopamine neurons exhibit electrical firing patterns and pharmacologic responses to dopamine agonists similar to the intrinsic dopamine cells of the substantia nigra pars compacta of adult animals [21].

Prior to transplanting human embryonic dopamine neurons into patients, human cells were transplanted into the rat model of Parkinson's disease. In cyclosporine immunosuppressed rats, human fetal dopamine neurons were shown to survive and produce behavioral effects equivalent to rat dopamine cells, albeit with a time course of 8 to 20 weeks compared to the 4 to 6 weeks seen with the faster developing rat neurites $[22,23]$. Xenograft transplants of the human to rat predicted the future difficulty of xenograft transplants in humans. While allografts of human embryonic dopamine neurons are not rejected following transplant, even with no immunosuppression [7], xenograft transplants of human into rat require continuous treatment with cyclosporine.

A pharmacologic misadventure by drug addicts has provided a primate model of Parkinson's disease. Initially, a young man trying to produce synthetic narcotics inadvertently injected himself with a mixture that was subsequently shown to include the neurotoxin 1-methyl-4phenyl-1, 2, 3, 6-tetrahydropyridine (MPTP) [24]. Shortly thereafter, he developed bradykinetic signs that were initially diagnosed as catatonic schizophrenia, but later as Parkinsonism. Because he was a single case, and the phenomenon could not be reproduced in rats, the report gained little attention. Had the investigators picked any other mammal, such as mouse, cat, dog, or monkey, they would have seen a Parkinsonian syndrome. Instead, this critical observation had to be rediscovered after a group of drug addicts got MPTP from a single dealer [25].

With the rediscovery of MPTP, a nonhuman primate model of Parkinson's was developed [26]. Monkeys lesioned by MPTP showed signs of bradykinesia, rigidity, and tremor with dopamine depletion in the caudate and putamen. L-DOPA improved these signs. Although the systemically-lesioned monkey closely resembled idiopathic Parkinson's disease, the condition was highly variable. Some animals recovered to normal, making it difficult to distinguish a therapeutic intervention from spontaneous improvement. Others, unable to feed themselves or maintain an upright posture, died of the complications of immobility. A more predictable lesioning method was developed by Bankiewicz et al. [27]. By unilaterally infusing MPTP into the internal carotid artery, dopamine neurons on one side of brain could be destroyed, leading to contralateral signs of Parkinsonism. This monkey model has the same characteristics as the unilaterally 6-OHDA-lesioned rat, a severe dopamine lesion on one side of the brain, but with normal dopamine levels on the other side of brain, making it possible for these animals to care for themselves.

Transplanting fetal dopamine neurons into denervated striatum reduced the Parkinsonian signs in both systemically and unilaterally lesioned monkeys, if the embryonic dopamine cells were obtained from early in embryogenesis at a developmental stage equivalent to days 13 to 16 in the embryonic rat [28-31]. By contrast, if mesencephalic tissue from later fetal stages was transplanted, cells failed to survive [32]. Monkey experiments were valuable for scaling up surgery to a larger brain, which had humanlike striatum divided into caudate and putamen. Monkey studies showed that the principles established in rats also applied to primates. Because embryonic monkey tissue of the appropriate early gestational stage is even more difficult to obtain than human fetal tissue, the number of monkey transplants reported in the literature has been small.

The question of targeting transplants to putamen, caudate, or both, is based on electrophysiological and transplant studies in monkeys. Alexander and DeLong [33] used microstimulation of the caudate and putamen to show that the putamen had motor responses specific to parts of the body with a homunculus that resembled that of motor and sensorimotor cortex. Leg and trunk movements were dorsolateral, arm movements were intermediate, and orofacial movements were ventromedial. By contrast, microstimulation of caudate produced no limb movements. Hikosaka et al. [34] performed a series of experiments in monkeys showing that caudate controls saccadic eye movements [34]. They also showed that caudate has a role in more complex behavioral responses to reward, perhaps reflecting its connections with prefrontal cortex [35]. The same group has found that monkeys with dopamine 
depletion of caudate show impaired eye movements, similar to patients with Parkinson's disease, but no abnormalities of limb movement [36].

A study in marmoset has directly compared transplants into caudate versus putamen. In the marmoset, dopamine neurons placed in caudate reversed amphetamine-induced circling, whereas transplants introduced into putamen improved motor function of the contralateral limb [37]. Taken together, these experiments in monkeys indicate that dopamine replacement in the putamen will directly improve movement of the limbs, whereas dopamine cell transplants into the caudate may have effects on eye movements and complex motor behaviors.

\section{Human Fetal Dopamine Cell Transplantation into Patients}

Since transplantation of human fetal tissue was first undertaken in the late 1980s, several groups have reported results using a variety of methods [3-7, 9, 38-45]. Comparisons among studies have been difficult because technical strategies and clinical evaluations have differed so widely. Tissue has been put into putamen, caudate, and substantia nigra with placement unilaterally and bilaterally. Transplants have been done as solid tissue fragments, as well as suspensions.

Another problem is the underlying variability of patients with Parkinson's disease. The variability in patient responses to L-DOPA points out that not all signs of disease are improved by dopamine replacement and that motor signs improve to a greater or lesser degree in individuals. Age is an important variable, as is the specific disease in the individual patient. To define patients as having idiopathic Parkinson's disease, most investigators chose patients who were responsive to L-DOPA. Some investigators have used fluorodopa (FDOPA) positron emission tomography (PET) to image the characteristic pattern of severe dopamine depletion in putamen with relative sparing in the caudate nucleus. No single clinical rating scale has been used, although there was an effort to define an evaluation strategy using a combination of the Unified Parkinson's Disease Rating Scale (UPDRS) and timed tests called Core Assessment Program for Intracerebral Transplantations (CAPIT) [46].

\section{Unilateral Versus Bilateral Transplantation}

Unilateral transplantation of fetal tissue was used in the first human clinical Parkinson's disease transplant trials [3, 4, 38]. Typically implants were made into the side of brain contralateral to the side of the body with the worse Parkinson's symptoms. In our Colorado experience, the first patient was transplanted with mesencephalic tissue from a single embryo injected in 1-cm long tracks with 6 needle passes into putamen and 4 needle passes into caudate via a vertex approach. Each needle pass was separated by $4 \mathrm{~mm}$ from other tracks.

Within 5 months, it was obvious that the unilateral transplant had improved motor performance only contralateral to the side of transplant [3]. Therefore, beginning with our third subject, we have performed bilateral, simultaneous implantation of fetal dopamine neurons into the putamen. Starting with our eighteenth open trial subject and prior to the double-blind NIH study, we switched from a vertex approach to a horizontal method using twist drill holes through the skull of the forehead above the frontal sinuses. The goal was to make the operation safer by reducing the number of needle passes from 8 needle penetrations of putamen per side to 2 needle passes per side by threading needles along the long axis of putamen in tracks approximately $4 \mathrm{~cm}$ in length. This unique neurosurgical approach has had the objective of providing bilaterally symmetric innervation of putamen and symmetrical improvement in motor function with a low risk of brain hemorrhage.

\section{Solid Versus Suspension Grafts}

Fetal mesencephalic tissue can be transplanted, either as a suspension of dissociated mesencephalon or as a solid graft. In some studies, human fetal tissue transplanted into immunosuppressed 6-OHDA-lesioned rats showed comparable survival of both solid and suspension grafts [47]. However, our studies showed that rat mesencephalon transplanted in the form of a strand (made by extruding mesencephalic tissue through a tapered glass cannula with a 0.2-mm bore) into 6-OHDA-lesioned rats produced greater behavioral improvement and better dopamine neuron survival than transplants of dissociated mesencephalon [48]. We have used this extrusion technique to create strands of tissue for human transplantation.

Immunology of Transplants

Because transplants of allografts in outbred rats and monkeys are not ordinarily rejected, even without immunosuppression, the need for immunosuppression in humans has been uncertain. Beginning with our first human transplant in 1988, we took the position that immunosuppression may not be necessary [3]. Because transplants of fetal dopamine neurons offer the potential for only symptomatic relief of the of Parkinson's disease symptoms, and because immunosuppression is expensive and carries substantial long-term medical risks, we decided not to immunosuppress our first subject [3]. After that we used immunosuppression in 6 of our first 12 transplant patients 
and found that immunosuppression did not improve transplant outcome [5]. Therefore, we did not use immunosuppression in our double-blind transplant study [7]. The Lund group chose to use immunosuppressants at doses equivalent to those given to kidney transplant patients [4]. When Freeman et al. [43] and Olanow et al. [9] started their transplant program approximately 5 years after our group and the Lund group, they took a middle ground, using short-term immunosuppression of 6 to 18 months. Our results after fetal dopamine cell transplants of monkey tissue into monkeys and human tissue into humans have shown no evidence of humoral or cellular immune responses to the transplanted tissue [45]. On the other hand, in 1 monkey we did observe apparent functional rejection of a previously well-established transplant 1 week following a second transplant of monkey mesencephalon into the opposite putamen. The tissue sample came from the same breeding colony as the first, with the same father, but a different mother [29]. Therefore, the apparent tolerance to allografts of fetal mesencephalic tissue may be only if transplants are done on a single occasion.

\section{Postmortem Survival of Human Fetal Dopamine Neurons}

Kordower et al. [42], who did bilateral transplants of fragments of embryonic mesencephalon into postcommissural putamen, were the first to demonstrate surviving dopamine cells postmortem. The patient was immunosuppressed with cyclosporine for 6 months and died 18 months after transplant. Large numbers of surviving dopamine neurons were seen in the transplant tracks. This result shows that dopamine neurons do not require continuous immunosuppression for long-term survival.

\section{Limitations of Nonblinded Clinical Trials for Clinical Assessments of Human Transplants}

Nearly all reports of human fetal dopamine cell transplants have had small groups of patients, changing methodology, and no placebo surgery controls. Statistically valid group comparisons have not been possible. Therefore, most reports have been anecdotal descriptions. Only the 2 NIH-sponsored clinical trials have had experimental designs with enough patients, consistent methodology, and control groups to make it possible to draw conclusions about clinical outcomes.

\section{Colorado - Columbia - North Shore University Double- Blind Trial}

\section{University of Colorado Transplant Methods}

Our double-blind, placebo controlled trial transplanted dopamine neurons from ventral mesencephalon recovered from 4 embryos at 7 to 8 weeks postconception and transplanted into 4 sites in the putamen, bilaterally under stereotaxic control during a single surgery. Tissue had been extruded through a tapered glass cannula into 200-micron strands and kept in culture for as long as 4 weeks prior to transplant, as previously described [5, 7]. Dopamine production was monitored on a continuous basis by assay of the dopamine metabolite homovanilic acid in the culture supernatant. Our center is the only one that tests every fetal ventral mesencephalon to show that each tissue is producing dopamine at a satisfactory level prior to transplant. Specimens that produce insufficient dopamine are not implanted [5, 7]. Dopamine neurons comprise a small percentage of the total neurons and glia of the ventral mesencephalon, and our measurements of dopamine production in tissue culture have shown that the output of dopamine was stable for at least 4 weeks. Although it would be desirable to know how other populations of neurons and glia evolve in culture, it has not been possible to inventory all cell types in these tissue strands.

After tissue from a single embryonic mesencephalon was proven to have adequate dopamine production, then that tissue fragment was taken from the culture and re-extruded into a 200-micron diameter strand in the operating room. Patients were awake during surgery, and needle penetrations were made via twist drill holes in the forehead under local anesthesia. The strand was drawn into the lumen of a needle with a bore of approximately 200 microns and injected into the putamen along its full horizontal length, a distance of approximately 30 to $40 \mathrm{~mm}$, using a continuous injection technique. The first embryonic tissue was always transplanted into the left putamen in a ventral position. The second embryonic tissue was transplanted $6 \mathrm{~mm}$ dorsal to the first transplant, and the third and fourth tissues were implanted into the right putamen in the same ventral and dorsal horizontal planes. Each transplant site required approximately 15 minutes from the twist drill hole to the completed infusion for a total of 60 minutes for the 4transplant procedure.

Sham surgery patients received the identical procedure with 4 twist drill holes in the forehead made over 60 minutes, except there was no needle penetration of the brain and no tissue was deposited. We, along with our institutional review board judged it was unethical to do needle passes into the brain of sham surgery patients. Studies in animal models of Parkinson's disease have shown that needle penetrations of the brain have no effect on either dopamine neurite outgrowth or motor behavior. Subjecting sham surgery patients to needle penetration of the brain would risk brain hemorrhage with no possibility of therapeutic benefit. All patients gave informed consent to receive either tissue transplants or sham surgery. For each patient, the blind was broken 1 year after transplant, after 
final clinical and PET scan results had been obtained. Sham surgery patients then had the opportunity to receive transplants. There were 14 of 20 who chose that option and received implants using the same transplant protocol that was used in the double-blind phase. Thus, a total of 34 of 40 subjects eventually received transplants.

All recovery of embryonic tissue from elective abortions was done only after women had consented to the abortion, and after they had given a second consent to donate fetal tissue per federal law and institutional review board requirements. There were no surgical complications that required breaking the blind in any subject. There were no infections.

\section{Clinical Effects of Transplants}

We found that 12 months after surgery, transplant subjects showed improvements in UPDRS motor "off" scores for the group as a whole and for the subgroup less than 60 years of age compared to the sham surgery controls (See Fig. 1) [7]. As shown in Fig. 2, transplants were detectable in $85 \%$ of patients by blind scoring of FDOPA PET scans and grew equally well in younger and older patients [49]. Those patients who were less than 60 years of age were most likely to benefit, and changes in UPDRS scores correlated with changes in the PET signal. The signs that improved were bradykinesia and rigidity. Although the tremor showed a trend for improvement, statistical significance was not reached. For each patient, the "best on" state was carefully determined after the first morning dose of LDOPA. After transplant, there was no change in the "best on" state. The sham surgery group had no changes in UPDRS motor or Schwab and England scores after surgery [7], indicating that there were no objectively measured placebo effects.

In patients with the best clinical responses, transplants could equal but not exceed the best effect of L-DOPA seen preoperatively. Therefore, transplants generate a purely dopaminergic effect. Just as with deep brain stimulation, Parkinsonian signs not improved by L-DOPA cannot be altered with transplants. Because transplants were placed only into putamen, we can say with some confidence that the motor effects of L-DOPA are mediated through actions on the putamen.

The primary outcome variable in our study, a global rating scale, proved to be a meaningless measure. While the study was still blinded at 12 months post-transplant, the patients were asked to pick terms that described their current state compared to 1 year before. Those descriptors were then assigned whole number values and averaged, as though they were continuous variables. Scores were recorded, and these patient recollections were used as the defining measure of outcome. Patients were then shown videos of themselves 1 year before, and all groups changed their ratings to more positive values. Our study demonstrated that a global rating scale cannot provide reliable assessments of long-term outcome in Parkinson's patients and should not be used.

Preoperative Response to L-DOPA is the Most Important Predictor of Transplant Outcome Regardless of Age

Because all subjects are now more than 10 years posttransplant, long-term analysis is possible. 18F-FDOPA PET scans have shown that transplants develop during a 2-year period and are stable at 4 years after transplant [50]. As previously noted, the most important predictor of response to transplant was the magnitude of the preoperative response to L-DOPA. Patients who had less than 50\% improvement after the first morning dose of L-DOPA did not respond to transplant. In our group of subjects, L-DOPA had a much broader range of effects in those who were older than 60 years of age compared to younger. Some older patients had only the minimal $30 \%$ improvement in UPDRS scores required to enter the study, whereas others had as much as a $90 \%$ improvement. Preoperatively, subjects who were less than 60 years of age had an average of $79 \%$ improvement after L-DOPA, whereas those who were older than 60 years of age had only 54\% improvement. That difference in the preoperative response to LDOPA fully accounts for the difference in transplant
Fig. 1 Changes in Unified Parkinson's Disease Rating Scale (UPDRS) motor "off" scores in the first 12 months after transplant during the double-blind phase. Results show significant improvement for the transplant group as a whole and for the younger transplant patients, as compared to the sham surgery group
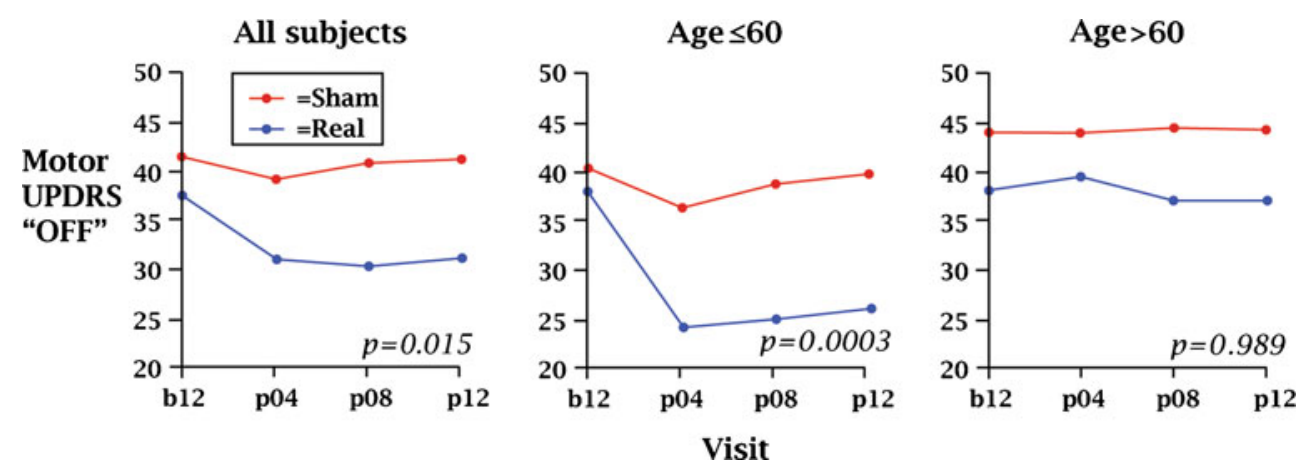
Embryonic Dopamine-Neuron Transplantation

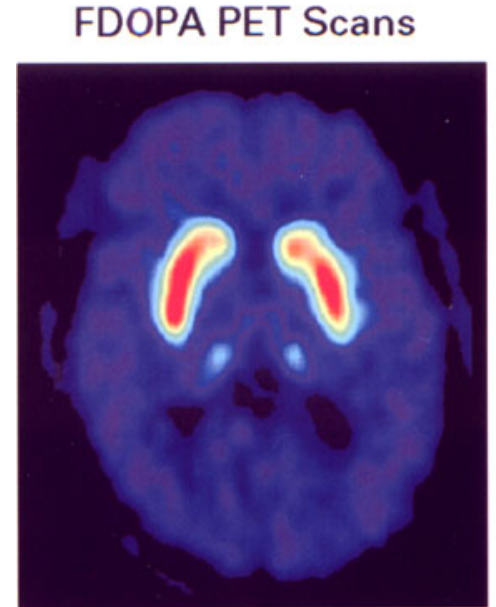

Normal

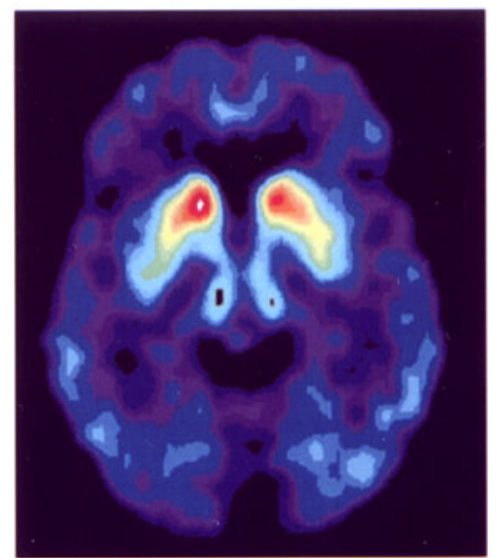

Before surgery

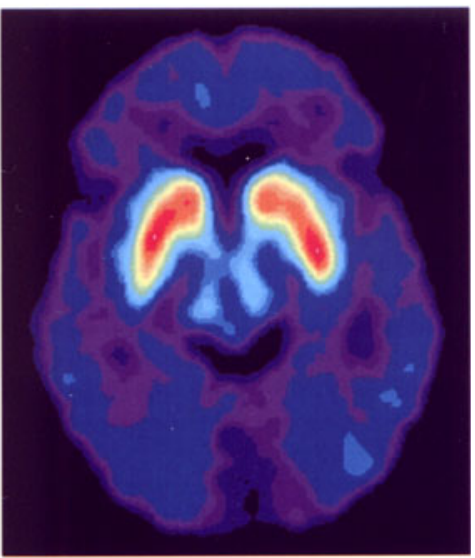

After surgery

\section{Sham Surgery}

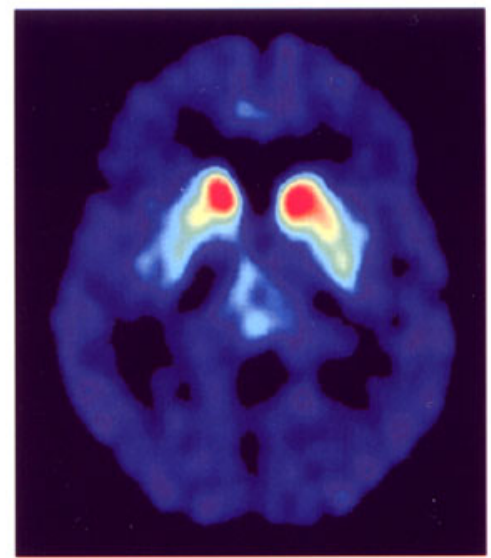

Before surgery

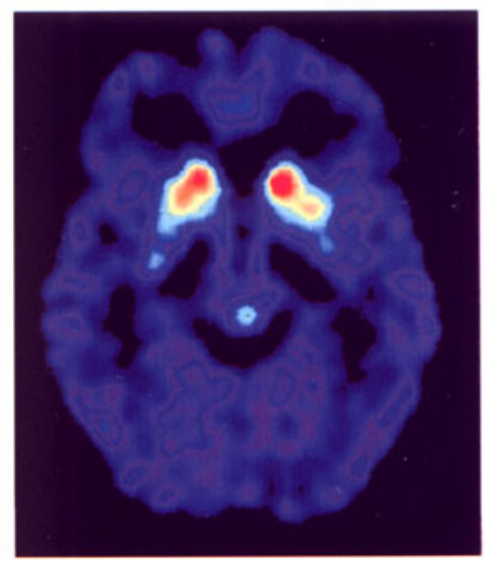

After surgery
Fig. 2 Fluorodopa (FDOPA) positron emission tomography (PET) scans before and after transplantation adapted from Freed et al. [7]. The left panel shows the FDOPA PET signal of a normal person scanned in a horizontal plane that includes caudate and putamen. Stored 18F-fluorodopamine is shown in the false color red. The 2 upper right panels show PET scans in a patient before and 12 months after implantation with embryonic dopamine neurons. The preoperative panel demonstrates the profound depletion of putamenal signal.

outcome for the two age groups. We have found that only subjects who have excellent clinical improvement preoperatively with L-DOPA will have a positive response after dopamine neurotransplantation.

When corrected for preoperative L-DOPA responsiveness, the older and younger patients had similar transplant outcomes. By 2 or 3 years after transplantation, UPDRS motor "off" scores showed that the transplant had replaced approximately $50 \%$ of the LDOPA requirement for both younger and older patients. Therefore, at the current stage of development, the average response to transplant is equivalent to approximately half of the L-DOPA effect. These long-term
The caudate signal is relatively higher. Twelve months after transplant, the FDOPA PET signal in putamen has increased toward the normal range. The 2 lower right panels show PET signals in a sham surgery patient before and 12 months after transplant. As shown, there is no improvement in putamenal dopamine. The caudate dopamine is further depleted. Reproduced with permission of The New England Journal of Medicine

changes are similar to what we first described in 1992 [5], and they are similar to what other groups have subsequently reported.

We would argue that a transplant that produces at least $50 \%$ of the best L-DOPA effect is an appropriate therapeutic goal. This effect is large enough to prevent severe "off" symptoms, while still not leading to dyskinetic movements [7]. With the transplant producing $50 \%$ of the needed dopamine, the optimum clinical response in the individual patient can be achieved by adding supplementary L-DOPA and other dopamine agonists.

Individual variability of transplant outcome is an important problem. Using tissue obtained from elective 
abortion, transplant outcome has varied from no effect whatsoever to complete replacement of the need for LDOPA. A primary goal for future transplants is to reduce the variability in individual patient outcome. Hopefully, stem cells differentiated in the laboratory to authentic midbrain dopamine neurons will provide a source of cells with more predictable growth and survival characteristics that will provide a more uniform clinical result. Cell transplantation that reliably replaces 50 to $80 \%$ of the LDOPA requirement in the individual patient could be an important component of dopamine replacement therapy for patients with Parkinson's disease.

Transplants that Replace the Need for L-DOPA

Can Replicate Drug-Induced Dyskinesias

in Susceptible Patients

Dyskinesias have appeared in transplant recipients who had LDOPA-induced dyskinesias prior to transplant $[3,5,7,9,48-$ 52]. In nearly all patients who have responded to transplants, there is an initial period of increased dyskinesias, which resolves as drug doses are reduced. In some patients, dyskinetic responses have persisted, even after substantial reduction or elimination of L-DOPA and other dopamine agonist therapies. In our double-blind study, all patients who developed persistent "off" dyskinesias were from the younger transplant group, and were also all who had L-DOPA-induced dyskinesias prior to transplantation [7, 51]. In those subjects, transplantation of tissue from 4 embryos produced enough dopamine to replace the need for L-DOPA and to replicate the dyskinesias previously seen with L-DOPA treatment. In the first year after transplant, these patients had experienced remarkable resolution of their parkinsonian signs. FDOPA PET scans showed some asymmetry of dopamine fiber outgrowth in these patients, with greater signal in the left ventral putamen than was seen in other patients who did not develop dyskinesias [51]. Patients did not show supranormal concentrations of dopamine.

Although asymmetric transplant growth may be an explanation for dyskinesias, it is more likely that the dyskinetic response seen after dopamine replacement with L-DOPA or transplantation is caused by abnormalities in the anatomic circuits in the dopamine-denervated Parkinsonian basal ganglia. These pathologic circuits have been shown to develop in striatum of dopamine-denervated animals, which show a loss of dendritic spines in medium spiny GABA-ergic projection neurons. With genetic knockout strategies, subpopulations of these medium spiny neurons have been shown to mediate different behavioral effects [53]. Differential damage to medium spiny neurons may account for the dyskinetic response to L-DOPA seen in patients with Parkinson's disease. There is no evidence that transplanted dopamine neurons are causing de novo dyskinesias.
We found a tendency toward dyskinetic movements in our first transplant recipient in 1990 [3], as well as in 6 of 7 patients we described in 1992 [5]. In most of these patients, dyskinesias were controlled by reductions in drug doses.

Neurophysiologic Testing Showed Improved Motor Function and No Effects on Cognitive Function

Neurophysiologic and cognitive effects of transplant were evaluated during the double-blind phase. Results showed significant improvement in reaction plus movement times [54]. This benefit was seen in both younger and older subjects. Cognitive performance showed no change in any measure 1 year after transplant [55].

Number of Donors - Tissue from 4 Donors Provides Sufficient Dopamine Neurons to Reverse Parkinson's Disease Signs

The number of surviving dopaminergic neurons needed to improve motor function significantly in a patient with Parkinson's disease is unknown. The normal adult human substantia nigra contains approximately 500,000 dopamineproducing neurons [56]. Unfortunately, approximately 95\% of dopamine neurons die after transplantation of rat or human tissue. In human patients, no more than 20,000 to 25,000 dopamine cells survive from each embryo transplanted [7, 42]. Some investigators have argued that such a high rate of cell death requires that tissue from 6 or more donors be transplanted into each putamen to restore a complete complement of dopamine-producing neurons. The issue is widely debated. Because the symptoms of Parkinson's disease develop only after a 50\% loss of nigral neurons and a 60 to $80 \%$ reduction in striatal dopamine [57, 58], complete replacement of dopamine-producing neurons may not be required to improve motor skills and to reduce the need for L-DOPA.

We have counted the dopamine neurons that have survived in transplant tracks in the postmortem brain. In 2 patients with bilateral putaminal implants who had clinical improvement sufficient to discontinue all L-DOPA, the total number of surviving dopamine neurons was 30,000 to 40,000 [59]. The 2 individuals had received tissue from 2 (open trial subject) and 4 embryos (double-blind trial subject), respectively.

Age as a Predictor of Transplant Outcome

Although Parkinson's disease is primarily a disease of elderly people, some researchers have been hesitant to perform transplantation in older patients. Our doubleblind study specifically addressed the issue of age with half of the recruited patients who were 60 years of age 
or older and with half of the patients who were younger than 60 years of age. As previously noted, it was not age per se, but the preoperative response to L-DOPA that correlated with transplant outcome. In the aging brain, the symptoms of Parkinson's disease are likely to be a manifestation of a more global neuropathologic process.

\section{Subjective Primary Outcome Variables \\ Should Not Be Used for Evaluating Long-Term \\ Patient Outcome}

Our double-blind trial has provided the only evidence of improved clinical outcome in a controlled neurosurgical trial of fetal dopamine cell transplantation. Despite these positive results, our study was formally deemed "negative" because of the unfortunate choice of a primary outcome variable. Our Columbia colleagues had insisted that the primary outcome variable be the subjective statement by each patient as to how they felt 1 year after the surgery compared to before the surgery. This variable proved to be meaningless because patients could not recall what their condition had been before the surgery. We conclude that subjective variables have no value for measuring outcome in a long-term study of Parkinson's disease.

\section{Mount Sinai - University of South Florida - Rush University Double-Blind Trial}

\section{Methods}

The second double-blind study of fetal dopamine cell transplants was performed by Olanow et al. [9]. The goal of their study was to compare placebo surgery with 2 doses of tissue, a "low" dose of cells from 2 embryos and a "high" dose of tissue from as many as 8 embryos transplanted into the putamen bilaterally [9]. There were 12 subjects in each of 3 groups: 1) 4 embryos per side, 2) 1 embryo per side, and 3) placebo surgery. Tissue was implanted into postcommissural putamen bilaterally during 2 independent operations. Placebo subjects had a sham surgery without needle penetration of the brain. All subjects, including placebos, were immunosuppressed for 18 months. Blinded follow-up continued for 24 months. For a primary outcome variable, they chose a variable that had been positive in our study - that is, the change in UPDRS motor "off" scores.

Clinical Outcome - No Significant Improvement in UPDRS Motor “Off” Despite Improved 18F-FDOPA PET Scans

Surprisingly, clinical assessments demonstrated no improvement in UPDRS motor "off" scores for either tissue dose compared to placebo surgery at the 24-month blinded endpoint. Substratifying patients by disease severity revealed that the "milder" group receiving 8 tissues had a modest improvement in scores compared to the deterioration observed in all other groups. Transplant survival was not the problem, because both 19F-FDOPA PET scans and autopsy results showed survival of dopamine neurons. FDOPA PET scans showed greater reinnervation of the putamen with the "high" tissue dose compared to the "low" dose at 1 year after transplant, but similar reinnervation at 2 years after transplant. This result is compatible with animal studies which indicate that transplant growth occurs in response to signals generated by denervated striatum [17]. Presumably, the transplants from the "low" dose group continued to have axon arborization between year 1 and year 2 to supply portions of putamen inadequately innervated in the first year. By this mechanism, the FDOPA PET scans of the "low-dose" group caught up with the "high-dose" group by the second year.

\section{Dyskinesias Seen in Some Transplant Subjects in the "Off"} State

Olanow et al. [9] did note dyskinesias were present in the "off" state in a large proportion of their transplant subjects and in none of the sham surgery patients. Although the dyskinesias were of minor significance in most, some patients required the placement of deep brain stimulating electrodes in the subthalamic nucleus to control excess movements. Therefore, Olanow et al. [9] replicated our earlier observation that transplants could reproduce dyskinesias in patients with an earlier history of drug-induced dyskinesias.

\section{Poor Clinical Response Probably Related to Poor Preoperative L-DOPA Response}

The authors could not account for the failure of the transplants to have effects on UPDRS motor "off" scores. Because immunosuppression was discontinued before the end of the double-blind phase, they speculated that transplants might have been rejected after the drugs were stopped. This conjecture is unlikely, because this group has shown surviving dopamine neurons in patients who died during the double-blind trial as well as in earlier subjects [9, 42]. In addition, FDOPA PET scan signals did not fall as they would if transplant rejection had occurred. They did find that patients with more severe baseline signs (higher UPDRS "off" scores) failed to improve after transplant [9].

Based on our experience, we would suggest that patient selection may have been the problem with the Olanow et al. [9] study. As we have shown, only those patients who have a very good to excellent response to L-DOPA are likely to 
benefit from transplant [7]. In their subjects preoperatively, L-DOPA produced an average improvement of 57\% [9]. In our younger group of subjects who responded briskly to transplants, the average preoperative response to L-DOPA was $79 \%$ improvement. By contrast, our older group of subjects who did not show benefit from transplant as a group had only a $54 \%$ average improvement from LDOPA, very similar to the average group response of Olanow et al. [9].

\section{Human Dopamine Neurons Survive Indefinitely Without Immunosuppression}

Once fetal dopamine neurons have survived the initial days after transplant, they appear to last indefinitely $[42,50,59$, 60]. Human dopamine cells transplanted and examined postmortem more than a decade after transplant show extensive reinnervation in the putamen $[59,61,62,63]$. In a small percentage of cells in some centers [61, 62], but not others [63], cytoplasmic inclusions resembling Lewy bodies have been observed. Because the overall quality of the dopamine cell transplant and fiber outgrowth remains excellent, the significance of this rare observation is uncertain.

\section{Laboratory Generation of Human Dopamine Neurons from Stem Cells}

To comprehensively explore the value of dopamine cell transplantation for Parkinson's disease, a more reliable tissue source must be found. The most promising source of an unlimited supply of dopamine neurons is from differentiated human embryonic stem cells or induced pluripotent stem cells [64-67]. While strategies have differed in details, the basic concept has been to differentiate embryonic stem cells to neuroprogenitor cells, then expand that cell population, and finally guide differentiation to dopamine neurons using known differentiation factors, such as sonic hedgehog and FGF8.

We, along with others, have transplanted these cells into immunosuppressed, unilateral 6-OHDA-lesioned rat models of Parkinson's disease [68-70]. Although these reports indicate that human dopamine neurons can survive and modify circling behavior in rats, they also show that residual stem cells can overgrow and cause tumor formation. A variety of strategies have been proposed for positive selection of dopamine neurons and for negative selection of residual stem cells. The ideal markers for positive selection would be on the cell surface. Corin has been identified on the surface of dopamine cell precursors and antibodies to corin can isolate populations of dopamine precursors, which are co-expressing green fluorescent protein (GFP) under control of the otx 2 promoter [71]. This kind of dual selection has not been worked out for human dopamine neurons. If a homogeneous supply of dopamine neurons can be generated, cell replacement can be systematically studied in large groups of patients, better defining the potential of this therapeutic strategy.

\section{Summary}

Since 1988, neurotransplantation with embryonic mesencephalic dopamine neurons has been tried as a treatment for patients with advanced Parkinson's disease. Although transplant methods have differed substantially among centers, most reports have found some value to cells implanted into the putamen. Despite these optimistic observations, most double-blind studies have failed to show any objective benefit of cell transplantation or growth factor infusion, even after positive results in open clinical trials. Well-controlled double-blind studies are essential to establish benefit in any neurosurgical intervention for Parkinson's disease.

Our double-blind study of fetal dopamine neurons is the only controlled trial showing that UPDRS motor scores (bradykinesia and rigidity) can significantly improve in transplant patients compared to placebo controls [7]. Several principles have emerged from our trial. Bilateral transplantation into the putamen can be done safely during a single operation. Mesencephalic tissue from early in embryonic development, 7 to 8 weeks after conception, can survive and provide sustained improvement of FDOPA uptake in putamen in nearly all subjects, regardless of patient age and without immunosuppression. Transplants can improve motor performance as much as the best effects of L-DOPA preoperatively seen. The preoperative L-DOPA response and not patient age determines the likelihood of improvement after transplantation. Immunosuppression is not required. Dyskinesias are seen after transplant in patients who have L-DOPA-induced dyskinesias prior to transplant and in whom the transplants replaced the need for L-DOPA.

Although the clinical benefit in individual patients has made drug elimination possible, there is substantial variability in outcome. Contributing to this variability are differences in pathologic processes in individual patients, as well as in differences in dopamine neuron survival and outgrowth. Some patients with a prior history of L-DOPAinduced dyskinesias may have persistent dyskinesias even after the reduction or elimination of L-DOPA. These dyskinesias respond to inhibitors of dopamine synthesis, such as metyrosine, or to deep brain stimulation of pallidum or subthalamic nucleus [72]. We have shown that transplants cannot improve the best effects of L-DOPA. 
Therefore, dopamine cell neurotransplantation can be best characterized as producing a steady-state infusion of dopamine into the putamen.

\section{Future Prospects for Neural Transplantation}

To fully evaluate the effectiveness of dopamine cell transplants for Parkinson's disease, a uniform source of dopamine neurons must be developed. Differentiation of embryonic stem cells or induced pluripotent stem cells to dopamine neurons may produce an unlimited number of dopamine neurons for transplant. We have found that allografts of human fetal mesencephalon are not rejected, unlike most somatic tissue transplants. Therefore, donorspecific embryonic stem cells will probably not be required, although the immunogenicity of stem cell-derived dopamine neurons could be different from fetal mesencephalon. Because of the enormous immunologic differences between species, successful xenografts are improbable.

Results of our double-blind study indicate that transplants placed only into the putamen can produce beneficial motor effects, which can be equal to the best effects of LDOPA and can replace the need for that drug. Although restoring uniform anatomic integrity of the nigrostriatal dopamine system is a daunting challenge, transplantation has already demonstrated successful repair of the human Parkinson brain. Nonetheless, transplant effects are limited to the replacement of dopamine. Clinical benefits have been incomplete in most patients. Many patients with Parkinson's disease eventually develop cognitive deterioration. There is no treatment for the progressive, nondopaminergic signs of Parkinson's disease. The ideal treatment for Parkinson's would slow or stop the underlying disease and prevent the loss of dopamine neurons and other affected cell types at the earliest stage of the disease.

Because dyskinesias in patients with Parkinson's disease may be a consequence of secondary anatomical and physiological changes in the basal ganglia after a long period of dopamine denervation and L-DOPA treatment, a clinical trial of transplantation early in Parkinson's disease may show that transplantation prevents the development of L-DOPA-induced dyskinesias. We proposed such a study to the NIH in 2003, but the idea was rejected at that time.

Dopamine cell transplantation may improve to the point that the outcome in an individual patient can be predicted with confidence. If neurosurgical intervention can reliably replace at least $80 \%$ of the need for L-DOPA and other drugs, then performing transplants can be a cost-effective way to treat the motor symptoms of Parkinson's disease, relieving the burden of tightly scheduled drug administration. This is a realistic goal for cell transplant repair of the Parkinson brain.
Acknowledgment Preparation of this article was facilitated by a scholarly residency at the Rockefeller Foundation Bellagio Center awarded to C.R.F. The double-blind controlled clinical trial of the University of Colorado, Columbia University, and North Shore University Hospital was supported by a grant from the National Institutes of Health, NIH RO1 NS 32368 (C.R.F., PI). Full conflict of interest disclosure is available in the electronic supplementary material for this article.

\section{References}

1. Bjorklund A, Steveni U. Reconstruction of the nigrostriatal dopamine pathway by intracerebral nigral transplants. Brain Res 1979;177:555-560.

2. Perlow MJ, Freed WJ, Hoffer BJ, et al. Brain grafts reduce motor abnormalities produced by destruction of nigrostriatal dopamine system. Science 1979;204:555-560.

3. Freed CR, Breeze RE, Rosenberg NL, et al. Transplantation of human fetal dopamine cells for Parkinson's disease. Arch Neurol 1990;47:505-512.

4. Lindvall O, Brundin P, Widner H, et al. Grafts of fetal dopamine neurons survive and improve motor function in Parkinson's disease. Science 1990;247:574-577.

5. Freed CR, Breeze RE, Rosenberg NL, et al. Survival of implanted fetal dopamine cells and neurologic improvement 12 to 46 months after transplantation for Parkinson's disease. N Engl J Med 1992;327:1549-1555.

6. Björklund A, Dunnett S, Brundin P, et al. Neural transplantation for the treatment of Parkinson's disease.Lancet Neurol 2003;2:437-445.

7. Freed CR, Greene PE, Breeze RE, et al. Transplantation of embryonic dopamine neurons for severe Parkinson's disease. N Engl J Med 2001;344:710-719.

8. Cohen J, New fight over fetal tissue grafts. Science 1994;263:600-601.

9. Olanow CW, Goetz CG, Kordower JH, et al. A double-blind controlled trial of bilateral fetal nigral transplantation in Parkinson's disease. Ann Neurol 2003;54:403-414.

10. Lang AE, Gill S, Patel NK, et al. Randomized controlled trial of intraputamenal glial cell line-derived neurotrophic factor infusion in Parkinson Disease. Ann Neurol 2006;S59:459-466.

11. Marks WJ Jr, Bartus RT, Siffert J, et al. Gene delivery of AAV2neurturin for Parkinson's disease: a double-blind, randomised, controlled trial.Lancet Neurol 2010;9:1164-1172.

12. Goetz CG, Stebbins GT 3rd, Klawans HL, et al. United Parkinson Foundation Neurotransplantation Registry on adrenal medullary transplants: presurgical, and 1- and 2-year follow-up. Neurology 1991;41:1719-1722.

13. Schumacher JM, Ellias SA, Palmer EP, et al. Transplantation of embryonic porcine mesencephalic tissue in patients with PD. Neurology 2000;54:1042-1050.

14. Deacon T, Schumacher J, Dinsmore J, et al. Histological evidence of fetal pig neural cell survival after transplantation into a patient with Parkinson's disease. Nat Med 1997;3:350-353.

15. Stover NP, Bakay RA, Subramanian $T$, et al. Intrastriatal implantation of human retinal pigment epithelial cells attached to microcarriers in advanced Parkinson disease. Arch Neurol 2005;62:1833-1837.

16. Ungerstedt U, Arbuthoott CW. Quantitative recording of rotational behavior in rats after 6-hydroxy-dopamine lesions of the nigrostriatal dopamine system. Brain Res 1970;24:485-493.

17. Dunnett SB, Hernandez TO, Summerfield A, Jones GH, Arbuthnott G. Graft-derived recovery from 6-OHDA lesions: specificity of ventral mesencephalic graft tissues. Exp Brain Res 1988;71:411-424. 
18. Hudson JL, Bickford P, Johansson M, Hoffer BJ, Stromberg I. Target and neurotransmitter specificity of fetal central nervous system transplants: importance for functional reinnervation. J Neurosci 1994;14:283-290.

19. Mahalik TJ, Finger TE, Stromberg I, et al. Substantia nigra transplants into denervated srriatum of the rat: ultrastructure of graft and host interconnections. J Comp Neurol 1985;240:60-70.

20. Schmidt RH, Ingvar M, Lindvall O, et al. Functional activity of substantia nigra grafts reinnervating the striatum: neurotransmitter metabolism and (14C)-2-deoxy-D-glucose autoradiography. J. Neurochem 1982;38:737-748.

21. Wuerthele SM, Freed WJ, Olson L, et al. Effect of dopamine agonists and antagonists on the electrical activity of substantia nigra neurons transplanted into the lateral ventricle of the rat. Exp Brain Res 1981;44:1-10.

22. Brundin P, Nilsson OG, Strecker RE, Lindvall O, Astedt B, Bjorklund A. Behavioral effects of human fetal dopamine neurons grafted in a rat model of Parkinson's disease. Exp Brain Res 1986;65:235-240.

23. Stromberg I, Bygdeman M, Goldstein M, Seiger A, Olson L. Human fetal substantia nigra grafted to the dopamine-denervated striatum of immunosuppressed rats: evidence for functional reinnervation. Neurosci Lett 1986;71:271-276.

24. Davis GC, Williams AC, Markey SP, et al. Chronic Parkinsonism secondary to intravenous injection of meperidine analogues. Psychiatry Res 1979;1:249-254.

25. Langston JW, Ballard P, Tetrud JW, Irwin, I. Chronic parkinsonism in humans due to a product of meperidine-analog synthesis. Science 1983;219:979-980.

26. Burns RS, Chiueh CC, Markey SP, Ebert MH, Jacobowitz DM, Kopin IJ. A primate model of parkinsonism: selective destruction of dopaminergic neurons in the pars compacta of the substantia nigra by N-methyl-4-phenyl-1,2,3,6-tetrahydropyridine. Proc Natl Acad Sci U S A 1983;80:4546-4550.

27. Bankiewicz KS, Oldfield EH, Chiueh CC, Doppman JL, Jacobowitz DM, Kopin IJ. Hemiparkinsonism in monkeys after unilateral internal carotid artery infusion of 1-methyl-4-phenyl1,2,3,6-tetrahydropyridine (MPTP). Life Sci 1986;39:7-16.

28. Bakay RAE, Fiandaca MS, Barrow DL, et al. Preliminary report of the use of fetal tissue transplantation to correct MPTP-induced Parkinsonlike symptoms in primates. Appl Neurophysiol 1985;48:358-361.

29. Freed CR, Richards JB, Sabol KE, Reite ML. Fetal substantia nigra transplants lead to dopamine cell replacement and behavioral improvement in Bonnet monkeys with MPTP induced Parkinsonism. In: Pharmacology and Functional Regulation of Dopaminergic Neurons. Beart PM, Woodruff G, Jackson DM, eds. Macmillan Press, 1988:353-360.

30. Annett LE, Martel FL, Rogers DC, Ridley RM, Baker HF, Dunnett SB. Behavioral assessment of the effects of embryonic nigral grafts in marmosets with unilateral 6-OHDA lesions of the nigrostriatal pathway. Exp Neurol 1994;125:228-246.

31. Annett LE, Torres EM, Clarke DJ, et al. Survival of nigral grafts within the striatum of marmosets with 6-OHDA lesions depends critically on donor embryo age. Cell Transplant 1997;6:557-569.

32. Redmond DE, Sladek JR Jr, Roth RH, et al. Fetal neuronal grafts in monkeys given methylphenyltetrahydropyridine. Lancet 1986;8490:1125-1127.

33. Alexander GE, DeLong MR. Microstimulation of the primate neostriatum. II. Somatotopic organization of striatal microexcitable zones and their relation to neuronal response properties. $\mathrm{J}$ Neurophysiol 1985;53:1417-1430.

34. Hikosaka O, Sakamoto M, Usui S. Functional properties of monkey caudate neurons. I. Activities related to saccadic eye movements. J Neurophysiol 1989;61:780-798.

35. Hikosaka O, Sakamoto M, Usui S. Functional properties of monkey caudate neurons. III. Activities related to expectation of target and reward. J Neurophysiol 1989;61:814-832.
36. Kori A, Miyashita N, Kato M, Hikosaka O, Usui S, Matsumura $\mathrm{M}$. Eye movements in monkeys with local dopamine depletion in the caudate nucleus. II. Deficits in voluntary saccades. J Neurosci 1995;15:928-941.

37. Annett LE, Torres EM, Ridley RM, Baker HF, Dunnett SB. A comparison of the behavioural effects of embryonic nigral grafts in the caudate nucleus and in the putamen of marmosets with unilateral 6-OHDA lesions. Exp Brain Res 1995; 103:355-371.

38. Lindvall O, Rehncrona S, Brundin P, et al. Human fetal dopamine neurons grafted into the striatum in two patients with severe Parkinson's disease. Arch Neurol 1989;46:615-631.

39. Spencer DD, Robbins RJ, Naftojin F, et al. Unilateral transplantation of human fetal mesencephalic tissue into the caudate nucleus of patients with Parkinson's disease. N Engl J Med 1992;327:1541-1548.

40. Widner H, Tetrud J, Rehncrona S, et al. Bilateral fetal mesencephalic grafting in two patients with Parkinsonism induced by I methyl-4-phenyl-1,2,3,6-tetrahydropyridine (MPTP). N Engl J Med 1992;327:1556-1563.

41. Peschanski M, Defer C, N'Guyen JP, et al. Bilateral motor improvement and lateralization of L-dopa effect in two patients with Parkinson's disease following intrastriatal transplantation of foetal ventral mesencephalon. Brain 1994;117:487-499.

42. Kordower JH, Freeman TB, Snow BJ, et al. Neuropathological evidence of graft survival and striatal reinnervation after the transplantation of fetal mesencephalic tissue in a patient with Parkinson's disease. N Engl J Med 1995;332:1118-1124.

43. Freeman TB, Olanow CW, Hauser BA, et al. Bilateral fetal nigral transplantation into the postcommissural putamen in Parkinson's disease. Ann Neurol 1995;38:379-388.

44. Freed CR, Breeze RE, Schneck SA, et al. Fetal neural transplantation for Parkinson's disease. In: Rich RR, ed. Clinical Immunology, Principles and Practice. St. Louis: Mosby-Year Book, 1995:1677-1687

45. Mendez I, Dagher A, Hong M, Hebb A, Gaudet P, Law A, Weerasinghe S, King D, Desrosiers J, Darvesh S, Acorn T, Robertson H. Enhancement of survival of stored dopaminergic cells and promotion of graft survival by exposure of human fetal nigral tissue to glial cell line-derived neurotrophic factor in patients with Parkinson's disease. Report of two cases and technical considerations. J Neurosurg. 2000;92(5):863-9.

46. Langston JW, Widner H, Goetz CG, et al. Core assessment program for intracerebral transplantations (CAPIT). Mov Disord 1992;7:2-13.

47. Freeman TB, Sanberg PR, Nauert CM, et al. The influence of donor age on the survival of solid and suspension inrraparenchymal human embryonic nigral cells. Cell Transplant 1995;4;141154.

48. Clarkson ED, Zawada WM, Adams FS, et al. Strands of embryonic mesencephalic tissue show greater dopamine neuron survival and better behavioral improvement than cell suspensions after transplantation in parkinsonian rats. Brain Res 1998;806:6068.

49. Nakamura T, Dhawan V, Chaly T, et al. Blinded positron emission tomography study of dopamine cell implantation for Parkinson's disease. Ann Neurol 2001;50:181-187.

50. Ma Y, Tang C, Chaly T, et al. Dopamine cell implantation in Parkinson's disease: long-term clinical and (18)F-FDOPA PET outcomes. J Nucl Med 2010;51:7-15.

51. Ma Y, Feigin A, Dhawan V, et al. Dyskinesia after fetal cell transplantation for parkinsonism: a PET study. Ann Neurol 2002;52:628-634.

52. Hagell P, Piccini P, Bjorklund A, et al. Dyskinesias following neural transplantation in Parkinson's disease. Nat Neurosci 2002;5:627-628. 
53. Bateup HS, Santini E, Shen W, et al. Distinct subclasses of medium spiny neurons differentially regulate striatal motor behaviors. Proc Natl Acad Sci U S A 2010;107:14845-14850.

54. Gordon PH, Yu Q, Qualls C, et al. Reaction time and movement time after embryonic cell implantation in Parkinson disease. Arch Neurol 2004;61:858-861.

55. Trott CT, Fahn S, Greene P, et al. Cognition following bilateral implants of embryonic dopamine neurons in PD: a double blind study. Neurology 2003;60:1938-1943.

56. Pakkenberg B, Moller A, Cundersen HJ, et al. The absolute number of nerve cells in substantia nigra in normal subjects and in patients with Parkinson's disease estimated with unbiased stereological method. J Neurol Neurosurg Psychiatry 1991;54:30-33.

57. Bernheimer H, Birkmayer W, Hornykiewicz O, et al. Brain dopamine and the syndromes of Parkinson and Huntington's: clinical, morphological and neurochemical correlations. J Neurol Science 1973;20:415-455.

58. Kish SJ, Shannak K, Hornykiewicz O. Uneven pattern of dopamine loss in the striatum of patients with idiopathic Parkinson's disease. N Engl J Med 1988;318:876-880.

59. Freed CR, Zawada M, DeMasters BK, et al. Human embryonic dopamine neurons transplanted into Parkinson patients survive and improve motor function for at least 10 years without immunosuppression. Program No. 329.6. Abstract Viewer/Itinerary Planner. Washington, DC: Society for Neuroscience, 2005.

60. Zawada WM, Zastrow DJ, Clarkson ED, et al. Growth factors improve immediate survival of embryonic dopamine neurons after transplantation into rats. Brain Res 1998;786:96-103.

61. Kordower JH, Chu Y, Hauser RA, Freeman TB, Olanow CW. Lewy body-like pathology in long-term embryonic nigral transplants in Parkinson's disease. Nat Med 2008;14:504-506.

62. Li JY, Englund E, Holton JL, et al. Lewy bodies in grafted neurons in subjects with Parkinson's disease suggest host-to-graft disease propagation. Nat Med 2008;14:501-503.
63. Mendez I, Viñuela A, Astradsson A, et al. Dopamine neurons implanted into people with Parkinson's disease survive without pathology for 14 years. Nat Med 2008;14:507-509.

64. Buytaert-Hoefen KA, Alvarez E, Freed CR, Generation of tyrosine hydroxylase positive neurons from human embryonic stem cells after co-culture with cellular substrates and exposure to GDNF. Stem Cells 2004;22:669-674.

65. Perrier AL, Tabar V, Barberi T, et al. Derivation of midbrain dopamine neurons from human embryonic stem cells. Proc Natl Acad Sci 2004;101:12543-12548.

66. Yan Y, Yang D, Zarnowska ED, et al. Directed differentiation of dopaminergic neuronal subtypes from human embryonic stem cells. Stem Cells 2005;23:781-790.

67. Zhou W, Freed CR. Adenoviral gene delivery can reprogram human fibroblasts to induced pluripotent stem cells. Stem Cells 2009;27:2667-2674.

68. Roy NS, Cleren C, Singh SK, Yang L, Beal MF, Goldman SA. Functional engraftment of human ES cell-derived dopaminergic neurons enriched by coculture with telomerase-immortalized midbrain astrocytes. Nat Med 2006;12:1259-1268.

69. Chiba S, Lee YM, Zhou W, Freed CR. Noggin enhances dopamine neuron production from human embryonic stem cells and improves behavioral outcome after transplantation into Parkinsonian rats. Stem Cells 2008;26:2810-2820.

70. Hargus G, Cooper O, Deleidi M, et al. Differentiated Parkinson patient-derived induced pluripotent stem cells grow in the adult rodent brain and reduce motor asymmetry in Parkinsonian rats. Proc Natl Acad Sci U S A 2010;107:15921-15926.

71. Chung S, Moon JI, Leung A, et al. ES cell-derived renewable and functional midbrain dopaminergic progenitors. Proc Natl Acad Sci U S A 2011;108:9703-9708.

72. Greene PE, Fahn S, Eidelberg D, Bjugstad KB, Breeze RE, and Freed CR. Persistent dyskinesias as a complication of fetal tissue transplantation for the treatment of Parkinson's disease. Movement Disorders, 2011, in press. 